\title{
Innovative Klimafolgenanpassung als Chance für die mittelständische Wirtschaft
}

\author{
Rainer Voß, Frank Hartmann
}

\section{Zusammenfassung}

Mit seinen Herausforderungen in den Bereichen Klimaschutz und Klimafolgenanpassung ist der Klimawandel in den vergangenen Jahren weltweit immer mehr in den Fokus von Wissenschaft, Politik und Öffentlichkeit gerückt. Die bisherigen Arbeiten und Diskussionen haben zu zahlreichen Ergebnissen geführt, die von der Abschätzung von Klimafolgen für einzelne Regionen bis hin zum Aufzeigen von geeigneten Anpassungsstrategien in den unterschiedlichen Anpassungsbereichen reichen. Zahlreiche Umsetzungsmaßnahmen auf Bundes- und Landesebene sind auf den Weg gebracht worden, um Regionen bei der Bewältigung dieser Prozesse zu unterstützen. Was in der Forschung und in der umsetzungsorientierten Praxis bisher weitgehend unberücksichtigt geblieben ist, ist die Frage nach der Umsetzung der Anpassungsstrategien und -maßnahmen, die letztlich nur durch das Hervorbringen innovativer Leistungsangebote durch Unternehmen erfolgen kann. Damit ist auch eine gewisse Perspektiverweiterung verbunden, weg von der Betroffenheit einzelner Anpassungsbereiche hin zu der Frage, wie kleine und mittlere Unternehmen am Prozess der Klimafolgenanpassung partizipieren können, indem sie mit innovativen Leistungsangeboten Zukunftsmärkte und Geschäftsfelder entwickeln.

\section{Abstract}

Climate change with its challenges in the fields of protection and adaption has more and more become in the focus of science, policy and public during the last year's world wide. Discussions and investigations up to now led to numerous results reaching from the assessment of climate impacts for single regions up to the showing of suitable adaption strategies in different adoption fields. Numerous implementation measures have been started in order to support regions in facing the processes of climate change. However, broadly unconsidered has been the question of transferring adoption strategies and measures in to innovative supplies by firms. With this to some extent a change in perspective is connected, away from concernment of different adoption fields towards to the question how small and medium sized firms can participate in the process of climate adaption developing innovative products and processes and herewith new markets and business segments.

\section{Einleitung}

Die deutsche Wirtschaft ist sehr stark mittelständisch geprägt. Von den fast 3,6 Mio. Unternehmen haben 99,5\% unter 250 Beschäftigte. Insgesamt generieren diese Firmen 38 \% des Umsatzes der gewerblichen Wirtschaft und haben einen Anteil von $55 \%$ an den Beschäftigten in diesem Bereich (ifm bonn, 2009). Wachstum und Wettbewerbsfähigkeit der Wirtschaft hängen wesentlich davon $\mathrm{ab}$, dass sich immer wieder wachsende Nachfragen nach Produkten und Dienstleistungen der vielen kleinen und mittleren Unternehmen herausbilden und diese mit innovativen Leis- tungsangeboten darauf reagieren. Es ist deshalb eine anspruchsvolle strategische Aufgabe des Innovationsmanagements, das Entstehen und die Entwicklung von Zukunftsmärkten zu verfolgen, an deren Erschließung Mittelstandsunternehmen nachhaltig partizipieren können.

Solche Zukunftsmärkte lassen sich verallgemeinert nach bestimmten Kriterien charakterisieren, wie insbesondere:

- langfristig stabile Problemlagen großer gesellschaftlicher Gruppen bzw. der Gesamtgesellschaft (national, international, global), z. B. demografischer Wandel, Gesundheit, Ernährung, Umwelt), 
- großes Marktpotenzial mit wachsendem Marktvolumen,

- zunehmende Überschneidungen zwischen individuellen, wirtschaftlichen und gesellschaftspolitischen Interessen bezogen auf Problemlösungsbedarfe und -strategien,

- hohe Variabilität und Dynamik in der Nachfrage nach zunehmend komplexen Problemlösungen (Produkten, Verfahren und Dienstleistungen),

- zukunftsfähiges Problemlösungspotenzial in Forschungs- und Technologiefeldern als Basis für ständig neue und verbesserte Leistungsangebote der Unternehmen,

- Wachstum verläuft über Innovations- und Qualitätswettbewerb,

- großer Handlungsspielraum für die Marktteilnehmer, insbesondere für alte und neue Anbieter.

Seit etwa 10 Jahren zeichnet sich z. B. verstärkt ab, dass mit Prozessen der zunehmenden Alterung moderner Industriegesellschaften und des individuellen Alterns hier lebender Menschen (überwiegend bis ins höhere Alter aktiv und leistungsfähig) die Entwicklung eines Zukunftsmarktes für innovative Problemlösungen in vielen Lebensbereichen älterer Menschen und für die Daseinsvorsorge der Gesellschaft verbunden ist (Brandt et al. 2003). Nach Auffassung der Autoren lassen sich nun auch begründete Argumente dafür entwickeln, dass sich auch in Folge des bisherigen und noch zu erwartenden Klimawandels, der daraus resultierenden Anforderungen des Klimaschutzes, insbesondere aber aus den Erfordernissen der Anpassung an aktuelle und zukünftige Klimafolgen ebenfalls ein neuer Zukunftsmarkt für vielfältige innovative Leistungsangebote, gerade auch von mittelständischen Unternehmen, herauszubilden beginnt. Ein erster Blick auf die oben genannten Kriterien lässt bereits erahnen, dass sich im Bereich Klimafolgenanpassung in den nächsten Jahren und Jahrzehnten ein sogenanntes »window of opportunity « für wirtschaftlich nutzbare Chancen öffnen wird. Dies soll im Weiteren begründet werden.

Der Klimawandel ist längst nicht mehr nur Gegenstand naturwissenschaftlicher Forschung, sondern rangiert seit mehreren Jahren auf den vorderen Rängen der Themenliste von Politik und Verwaltung auf nahezu allen Ebenen und vielfältige Maßnahmen, wie z. B. bundesweite Wettbewerbe (Klima zwei, KLIMZUG und KLIWAS), werden ergriffen, um den Herausforderungen zu begegnen. Die Fragestellung, welche
Funktion kleine und mittlere Unternehmen bei der Klimafolgenanpassung haben und wie dies mit einer erfolgreichen Regionalentwicklung in Zusammenhang gebracht werden kann, ist zwar politisch artikuliert (Grünbuch 2007, S. 13), aber wissenschaftlich bisher kaum untersucht. Mit dem folgenden Beitrag soll ein Zugang zur Problematik des Klimawandels vorgestellt werden, der insbesondere auf regionalwissenschaftlichen und innovationstheoretischen Ansätzen beruht und mit dem erklärt werden kann, warum und wie insbesondere kleine und mittlere Unternehmen an den unterschiedlichsten Prozessen der Klimafolgenanpassung partizipieren können und welche Unterstützungsmaßnahmen hierfür in frühen Phasen der Marktentstehung sinnvoll entwickelt werden können.

\section{Klimafolgenanpassung}

Der Klimaschutz zielt hauptsächlich auf die Verringerung von Emissionen. Damit kann er dazu beitragen, die einerseits erforderlichen Zeiträume für die Anpassung an die Folgen des Klimawandels zu schaffen und den Anpassungsdruck soweit zu verringern, dass Anpassungsmaßnahmen überhaupt sinnvoll möglich werden. Nachhaltige Anpassungsmaßnahmen an die Folgen des Klimawandels müssen andererseits immer auch den Klimaschutz unterstützen. Beide stehen in einem engen Zusammenhang, verlangen aber auch spezifische Strategien und Maßnahmen für unterschiedliche Klimafolgenbereiche wie Wasserwirtschaft, Land- und Forstwirtschaft, Verkehr, Tourismus, Gesundheit, Raum- und Siedlungsentwicklung, Naturschutz und Biodiversität einschließlich der Wirtschaft. So wurden in den vergangenen Jahren basierend auf wissenschaftlicher Expertise und praktischen Erfahrungen Klimafolgen in ausgewählten Anpassungsbereichen und darauf ausgerichtete Anpassungsstrategien bzw. -maßnahmen herausgearbeitet (Zerbisch et al. 2005; BMU 2008; Schuchardt et al. 2008). Eine beispielhafte Übersicht dazu zeigt die Tabelle 1.

Bisherige Forschungsarbeiten zum Klimawandel thematisieren seine Ursachen, schätzen seine eher direkten Folgen (z. B. Temperatur, Niederschlag, Wind) auf Bereiche ab, die unmittelbar klimarelevant sind (Land- und Forstwirtschaft, Biodiversität, Gesundheit u. a.). Sie gehen noch einen Schritt weiter, indem sie die prinzipiellen Erfordernisse zur Anpassung an die- 


\begin{tabular}{|c|c|c|}
\hline $\begin{array}{l}\text { Anpassungs- } \\
\text { bereich }\end{array}$ & Klimafolgen & Anpassungsmaßnahmen \\
\hline $\begin{array}{l}\text { Wasserwirt- } \\
\text { schaft }\end{array}$ & $\begin{array}{l}\text { Gefahr von } \\
\text { Hochwasser }\end{array}$ & $\begin{array}{l}\text { Technischer Hochwasserschutz/ } \\
\text { Künstliche Rückhalte erhalten } \\
\text { und erweitern }\end{array}$ \\
\hline Landwirtschaft & $\begin{array}{l}\text { Trockenstress } \\
\text { im Sommer }\end{array}$ & $\begin{array}{l}\text { Angepasste Bewässerungs- } \\
\text { verfahren }\end{array}$ \\
\hline Forstwirtschaft & $\begin{array}{l}\text { Erhöhte Wald- } \\
\text { brandgefahr }\end{array}$ & Vorsorge gegen Waldbrände \\
\hline Gesundheit & $\begin{array}{l}\text { Mehr heiße } \\
\text { Sommertage }\end{array}$ & $\begin{array}{l}\text { Verbesserung der Kühlung für } \\
\text { besondere Personenkreise und } \\
\text { Gebäudetypen }\end{array}$ \\
\hline Tourismus & $\begin{array}{l}\text { Mehr heiße } \\
\text { Sommertage }\end{array}$ & Steigerung der Attraktivität \\
\hline $\begin{array}{l}\text { Verkehrs- } \\
\text { infrastruktur }\end{array}$ & $\begin{array}{l}\text { Extremwetter; } \\
\text { Hitze, Sturm, } \\
\text { Starkregen, } \\
\text { Eis }\end{array}$ & $\begin{array}{l}\text { Schutz von Straßen gegen } \\
\text { Extremereignisse }\end{array}$ \\
\hline $\begin{array}{l}\text { Bau- und } \\
\text { Siedlungs- } \\
\text { wesen }\end{array}$ & $\begin{array}{l}\text { Extremwetter, } \\
\text { Starkregen }\end{array}$ & $\begin{array}{l}\text { Niederschlagskontrolle, } \\
\text { verbesserte Dachentwässerung }\end{array}$ \\
\hline
\end{tabular}

Tabelle 1: Beispiele für Klimafolgen, Anpassungsbereiche und -maßnahmen

se Folgen aufzeigen und bewerten (Vulnerabilität und Anpassungskapazität) (Umweltbundesamt 2005). Dies erfolgt unter methodischen Gesichtspunkten ganz wesentlich auf der Basis von Modellen und Szenarien. Thematisiert wird auch die Betroffenheit bzw. die Anfälligkeit der Wirtschaft (z. B. in den Bereichen Energie, Wasser und Verkehr) von bzw. für Klimafolgen. Bisher kaum thematisiert werden dagegen Fragen nach den Akteuren und Akteurskonstellationen sowie nach den Bedingungen, unter denen diese auf verschiedene Folgen in definierten Regionen und bestimmten Bereichen mit Anpassung reagieren können. In dem hier zu entwickelnden Zugang geht es folglich auch darum, eine Perspektiverweiterung bezogen auf den Zusammenhang von Klimafolgen und Wirtschaft zu vollziehen, von der Betrachtung der Wirtschaft als ein von Folgen betroffener Bereich hin zur Betrachtung der Wirtschaft als ein Hauptakteur bei der Klimafolgenanpassung. In diesem Verständnis wäre danach zu fragen, wie Unternehmen mit wem innovative Lösungen entwickeln können, um die Anfälligkeit verschiedener gesellschaftlicher Bereiche, einschließlich der Wirtschaft, zu verringern und deren Anpassungskompetenz und -kapazität zu erhöhen.

\section{Klimafolgenanpassungskompetenz und Innovation}

Einen interessanten Ansatz stellt in diesem Zusammenhang die Rolle des Ressourcenaustausches zwischen Unternehmen und Unternehmensumwelten bei der Hervorbringung von Innovationen dar (Voß 1996). Entsprechend diesem Ansatz kombinieren Unternehmen im Prozess der Leistungserstellung unternehmensinterne und -externe Ressourcen, wobei sich die externen Ressourcen und die sie gestaltenden Akteure in den so genannten generellen Unternehmensumfeldern (vom ökologischen, makroökonomischen bis hin zum technologischen Umfeld) und spezifischen Unternehmensumfeldern (vom Arbeitsmarkt, über den Finanzmarkt bis hin zu Kunden und Zulieferern) verorten lassen. In den entsprechenden Ressourcenaustauschprozessen liegen die Möglichkeiten und auch die Notwendigkeiten für die Hervorbringung von Innovationen durch Unternehmen begründet: Sei es, dass sich Ressourcen verknappen, sich neue Kombinationsmöglichkeiten auftun oder mehrere Kombinationsprozesse im Wettbewerb stehen. Auch stammen Innovationsimpulse häufig aus diesen Unternehmensumfeldern, die in engen Wechselwirkungen stehen und auf unterschiedlich konditionierte Unternehmen wirken. Diese Unternehmensumfelder bzw. ihre Komponenten haben neben der funktional-inhaltlichen Dimension auch eine räumliche Dimension, weshalb die regionale Komponente für die Problematik des Innovierens von Unternehmen überhaupt erst Bedeutung erlangt. Zahlreiche Innovationsansätze der vergangenen Jahre gehen davon aus, dass räumliche Nähe Transaktionskosten senkend und/oder Vertrauen bildend wirken kann, was zu solchen Konzepten wie »Innovationsnetzwerke«, „Cluster « oder »regionale Innovationssysteme« geführt hat, so dass der Regionalbezug diesen Konzepten zwar immanent war, jedoch eher sekundär blieb, thematisiert durch das Zusammenspiel unterschiedlicher, an Innovationsprozessen von Unternehmen beteiligter regionaler Akteure. Viele dieser Ansätze lassen sich unter dem Oberbegriff »innovationsorientierte Regionalpolitik « zusammenfassen, in deren Kern es darum geht, die regionalen Bedingungen für das Innovieren von Unternehmen zu verbessern (Koschatzky 2002; Fritsch 2003; Porter 1998; Ellwein et al. 1982). Im Mittelpunkt dieser Ansätze stehen die an der Hervorbringung und Realisierung von Innovationen beteiligten Akteure, weniger die Region. In dieser 
Perspektive würden Klimawandel und Klimafolgenanpassung einhergehen mit Veränderungen in generellen und spezifischen Unternehmensumfeldern, die Innovationen herausfordern könnten, und je nachdem welche Ressourcen getauscht werden müssten und wie deren räumliche Ausprägung aussähe, regional hervorgebracht werden könnten oder auch nicht.

Einen Schritt weiter geht der Ansatz der so genannten »regionalorientierten Innovationspolitik«, in dessen Zentrum die Region als Subjekt steht, auf die sich die Innovationsaktivitäten der Unternehmen und anderer an der Hervorbringung und Durchsetzung von Innovationen beteiligter Akteure, vornehmlich aus der Region, richten (Voß 2005). In diesem Verständnis haben Regionen selbst einen Innovationsbedarf, der sich etwa im Erneuerungsbedarf einer einzelnen regionalen Entwicklungsbedingung (z. B. der Verkehrsinfrastruktur) äußern kann. Zugleich verfügen Regionen über endogene Potenziale, die in der Lage sind, auf diese Innovationsbedarfe durch die Hervorbringung von Innovationen zu reagieren (etwa im Bereich der regionalen Wirtschaft). In dieser Perspektive würden Klimawandel und Klimafolgenanpassung direkt mit der Regionalentwicklung in Zusammenhang gebracht, da diese direkt auf einzelne regionale Entwicklungsbedingungen (z. B. den regionalen Wasserhaushalt) wirken (negativ oder positiv) und damit das Innovationsbedarfspotenzial der Region erhöhen. Weil die regionalen Entwicklungsbedingungen unter zunehmenden Anpassungsdruck geraten, wird das regionale Innovieren stimuliert. Die Region erscheint zugleich als Voraussetzung (regionale Entwicklungsbedingungen sind für Innovationsprozesse erforderlich und haben darauf bezogen eine Versorgungsfunktion) und Ziel des Innovierens.

Besonders erfolgreiche Regionen sind als solche mit hoher Innovationskompetenz, die an der Schnittstelle von »Innovationspotenzial«, »Innovationsfähigkeit«, »Innovationsbereitschaft « und »Handlungsspielräume« entsteht und die Fähigkeit zur rechtzeitigen Wahrnehmung und Bewertung regional bedeutsamer Veränderungen in den Unternehmensumfeldern und zur Entwicklung darauf bezogener regionaler strategischer Innovationsansätze einschließt. Klimafolgenanpassungskompetenz wäre demzufolge eine spezifische Komponente einer so verstandenen regionalen Innovationskompetenz und könnte wie folgt im Grundzusammenhang von Klimawandel und Klimafolgenanpassung verortet werden (vgl. Abb. 1).

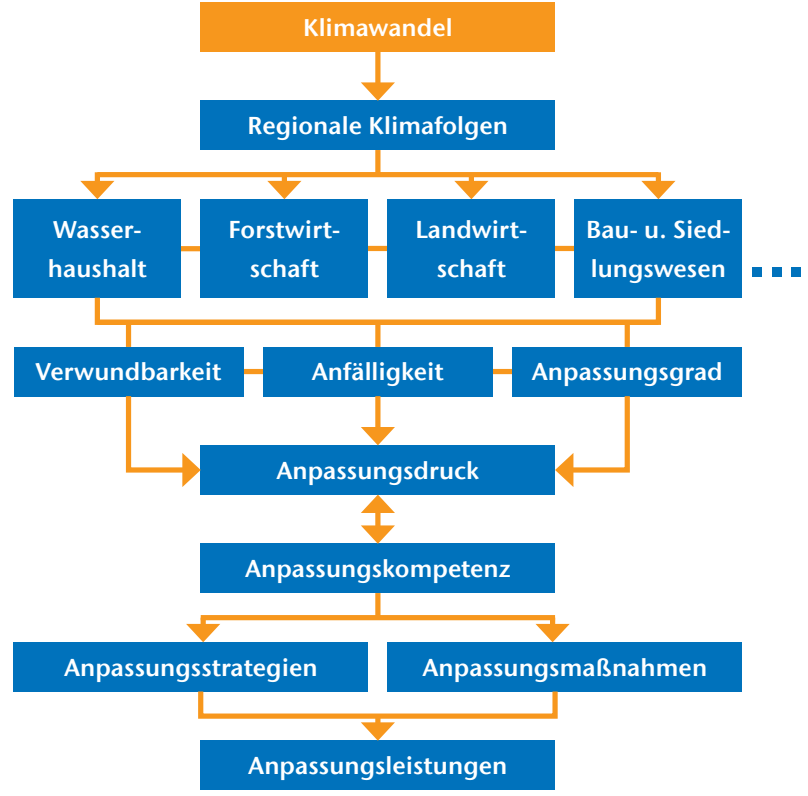

Abb. 1: Verortung der Klimafolgenanpassungskompetenz

Anpassungskompetenz wäre in diesem Verständnis die Fähigkeit zur Aufnahme des Anpassungsdrucks aus den verschiedenen Anpassungsbereichen und zur Transformation dieses Drucks in Anpassungsstrategien und -maßnahmen als strategische Orientierungsfelder für die Hervorbringung und Durchsetzung innovativer Anpassungsleistungen durch die Wirtschaft in einer Region. Auch diese Kompetenz entsteht aus der Integration von erforderlichem Wissen, Fähigkeiten und Erfahrungen, hoher Motivation/Bereitschaft und notwendigen Handlungsspielräumen, um

- Klimafolgen als gesamtgesellschaftliche, regionale, sektorale und personale Problemlagen in unterschiedlichen Folgenbereichen frühzeitig in ihrer Entstehung und möglichen Entwicklung wahrzunehmen,

- Klimafolgen hinsichtlich ihrer Ursachen, Abhängigkeiten und Wirkungszusammenhänge zu bewerten,

- Anpassungsstrategien und -maßnahmen sowie Anpassungsleistungen für die Vorbeugung, Verzögerung, Verminderung oder Vermeidung von Klimafolgen hervorzubringen und anzuwenden und

- dafür die erforderlichen Potenziale und Akteure zu mobilisieren. (vgl. Abb. 2).

Klimafolgenanpassungskompetenz wird permanent durch Informations-, Kommunikations-, Kooperationsund Vernetzungsaktivitäten (z. B. zwischen Unternehmen, wissenschaftlichen Einrichtungen, Kommunalverwaltungen, Intermediären, Behörden, Medien und Privatpersonen) entwickelt. Neben der Sensibilisierung von Unternehmen, Kommunen, Wissenschafts- und 


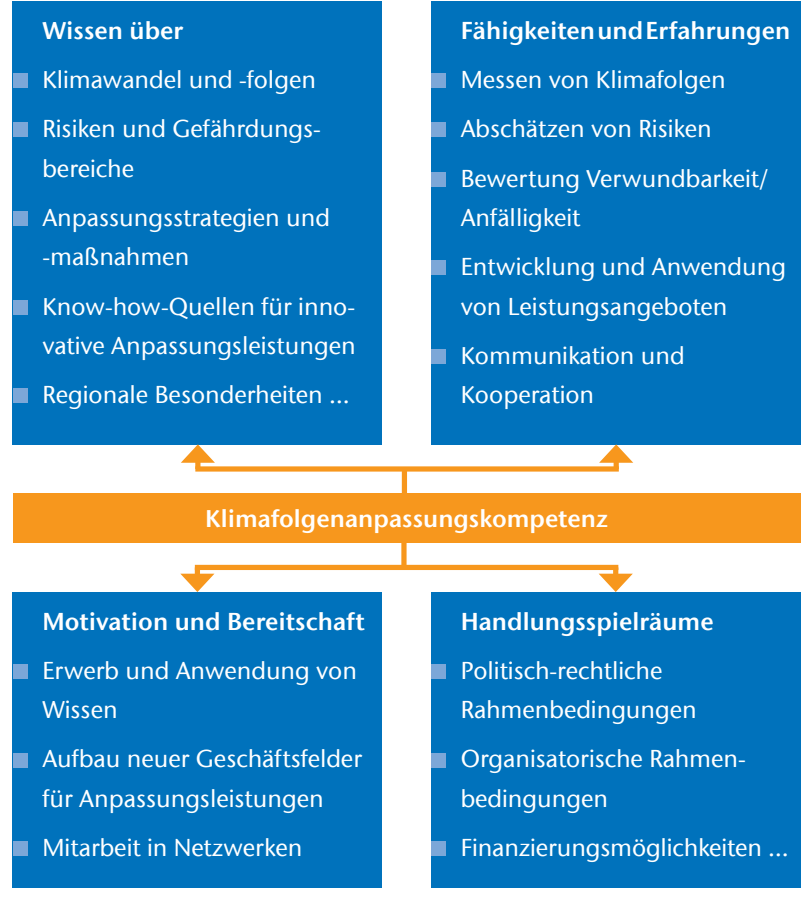

Abb. 2: Klimafolgenanpassungskompetenz

Bildungseinrichtungen, Verwaltung und Politik bis hin zu betroffenen Privatpersonen und der Erhöhung ihrer Anpassungskompetenz spielt der Aufbau entsprechender regionale Netzwerke eine Rolle, da ein solcher Prozess in der Regel nicht von einem einzelnen regionalen Akteur (Person oder Institution) zielführend und problemlösungsorientiert realisiert werden kann.

\section{Unternehmerische Leistungsangebote zur Klimafolgenanpassung und Suchraum für Geschäftsfelder}

Aufgrund des steigenden Anpassungsdrucks gilt für alle oben genannten Anpassungsbereiche, dass sie in den nächsten Jahren und Jahrzehnten Anpassungsmaßnahmen, basierend auf Anpassungsstrategien, entwickeln müssen (vgl. Abbildung 1). Daraus entstehen zunehmend Problemlösungsbedarfe für eine adäquate Anpassung an Klimafolgen und damit Bedarfe und Nachfragen nach Produkten, Verfahren und Dienstleistungen, welche die bereichsspezifischen Anpassungsstrategien und -maßnahmen ermöglichen bzw. unterstützen (Ott et al. 2008). Eine wesentliche Aufgabe besteht dabei darin, die Brücke von den Anpassungsstrategien zu den innovativer Leistungsangeboten der Wirtschaft $\mathrm{zu}$ schlagen und dies nicht nur konzeptionell, sondern auch praktisch, indem Unternehmen für die Thematik sensibilisiert und bei der Markterschließung unterstützt werden. Hierfür können verschiedene Innovationsmanagementansätze herangezogen werden.

Ausgewählte Beispiele für mögliche Leistungsangebote im Bereich der Anpassung an Klimafolgen sind in Tabelle 2 aufgeführt. Bereits diese wenigen Beispiele deuten auf eine große Vielfalt hin, lassen aber auch die Komplexität der entsprechenden Anwendungskontexte erahnen, die für entsprechende Leistungsangebote relevant sind.

Um sich der Hervorbringung und Vermarktung von Innovationen zur Klimafolgenanpassung systematischer zu nähern, können sich Unternehmen beim Aufbau von Geschäftsfeldern an einem speziellen »Suchraum« orientieren, der durch die Dimensionen »Klimafolgentyp«, »Anpassungsbereiche (Nachfrager)« und »Leistungsbereiche« gebildet wird (vgl. Abbildung 3). In diesen Raum können Unternehmen, aber auch andere Leistungsanbieter, »Schnitte« legen, um basierend auf ihren Kompetenzen neue Geschäftsfelder aufzubauen. Dieser Suchraum ist auch geeignet, Unternehmensgründungen gezielt am Markt zu platzieren. Auch für unternehmensübergreifende Aktivitäten im Bereich der Klimafolgenanpassung, etwa beim Aufbau entsprechender Netzwerke, kann dieser dreidimensionale Suchraum als heuristisches Element genutzt werden.

\begin{tabular}{|c|c|c|c|}
\hline Anpassungsbereich & Klimafolgen & Anpassungsmaßnahmen & Leistungsangebote \\
\hline Wasserwirtschaft & $\begin{array}{l}\text { Gefahr von } \\
\text { Hochwasser }\end{array}$ & $\begin{array}{l}\text { Technischer Hochwasserschutz/Künst- } \\
\text { liche Rückhalte erhalten und erweitern }\end{array}$ & $\begin{array}{l}\text { Einlaufbauwerke für gesteuerte Polder; neue Antriebs- } \\
\text { elemente im Stahlwasserbau }\end{array}$ \\
\hline Landwirtschaft & $\begin{array}{l}\text { Trockenstress } \\
\text { im Sommer }\end{array}$ & Angepasste Bewässerungsverfahren & $\begin{array}{l}\text { Automatisierte, wasser- und energiesparende } \\
\text { Beregnungsanlagen; Pumptechnik, Düsen... }\end{array}$ \\
\hline Gesundheit & $\begin{array}{l}\text { Mehr heiße } \\
\text { Sommertage }\end{array}$ & $\begin{array}{l}\text { Verbesserung der Kühlung für besondere } \\
\text { Personenkreise und Gebäudetypen }\end{array}$ & $\begin{array}{l}\text { Hybride Heiz- und Kühlsysteme; Baustoffe; Passivbau- } \\
\text { haus; Erdreichwärmetauscher für Gebäudekühlung; } \\
\text { Durchlüftbarkeit von Gebäuden; Dachbegrünung }\end{array}$ \\
\hline $\begin{array}{l}\text { Bau- und Siedlungs- } \\
\text { wesen }\end{array}$ & $\begin{array}{l}\text { Extremwetter, } \\
\text { Starkregen }\end{array}$ & $\begin{array}{l}\text { Niederschlagskontrolle, verbesserte } \\
\text { Dachentwässerung }\end{array}$ & $\begin{array}{l}\text { Mess- und Registriersysteme; Schutzauslöser; } \\
\text { neue Baumaterialien und -technologien; Dachrinnen }\end{array}$ \\
\hline
\end{tabular}

Tab. 2: Beispiele für mögliche Leistungsangebote zur Klimafolgenanpassung 


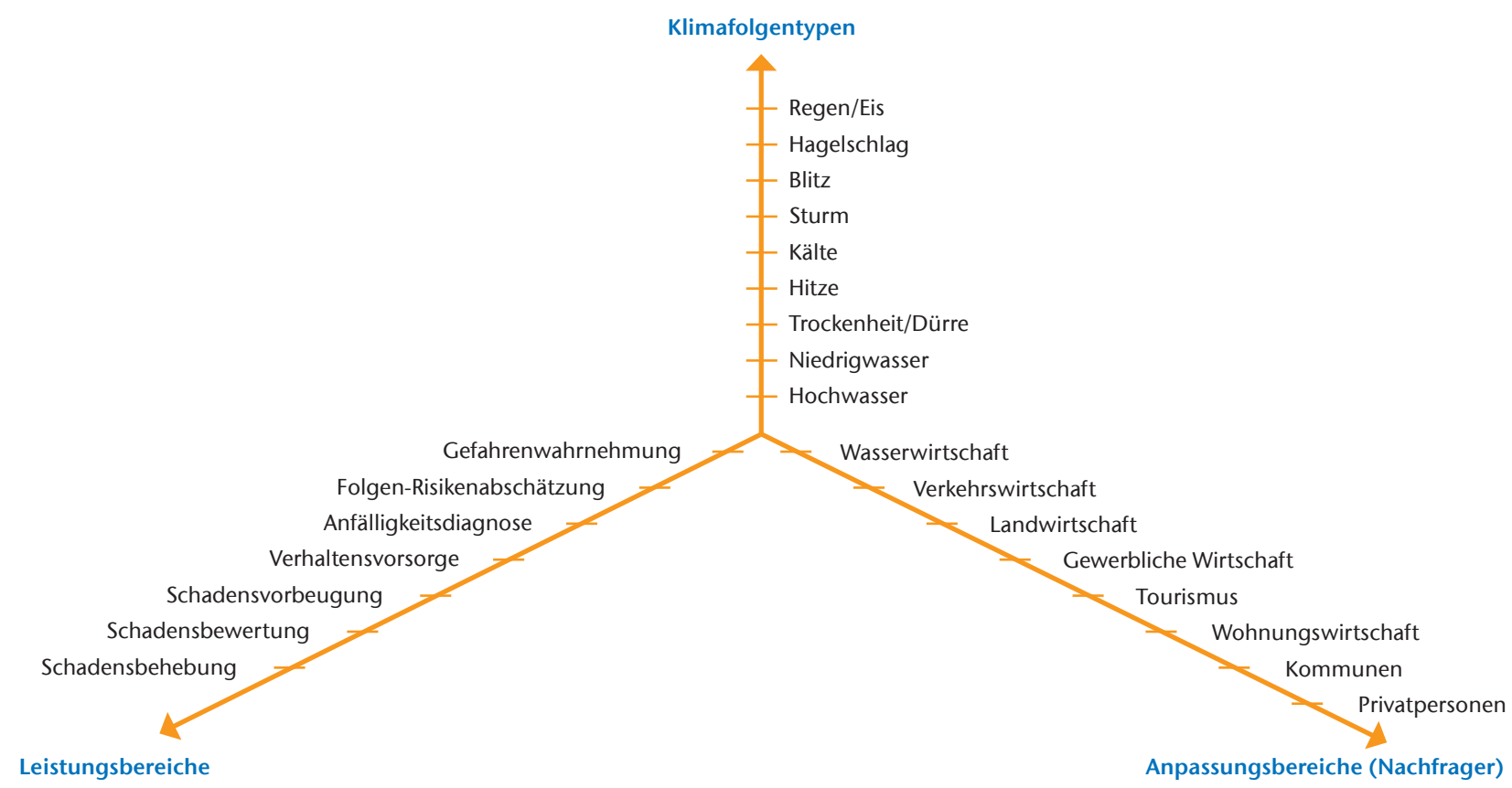

Abb. 3: Suchraum für mögliche Geschäftsfelder von KMU im Bereich Klimafolgenanpassung

Je nach Kompetenzen eines Unternehmens und die Entwicklungen im Unternehmensumfeld berücksichtigend können Unternehmen entlang der Dimension »Leistungsbereiche« bezogen auf einzelne Komponenten Geschäftsfelder aufbauen oder auch Komponenten übergreifend agieren. Gleiches trifft auf die anderen beiden Dimensionen »Klimafolgen « und »Nachfrager « zu (vgl. Abbildung 3).

Im Falle zu hoher Komplexität und zu großer technischer, marktlicher oder planungsseitiger Unsicherheiten bestimmter Geschäftsfelder für einzelne Unternehmen werden sich Netzwerke zwischen Unternehmen aufbauen. Bei der Entwicklung neuer Leistungsangebote dürften Innovationsnetzwerke eine Rolle spielen, in die auch Forschungseinrichtungen eingebunden sein werden.

Das Spektrum von Branchen die innovative Anpassungsleistungen hervorbringen, ist erheblich und reicht von der Energie- und Wasserversorgung über den Maschinen- und Anlagenbau, die Metallerzeugung und -bearbeitung, Informationstechnologie, Elektronik, Elektrotechnik, Sensorik, Messtechnik, Verkehr und Nachrichtenübermittlung, Automobil- und Luftfahrtindustrie, Bauwirtschaft (Hoch- und Tiefbau, Bauinstallation, Ausbau, Wasserbau, Straßenbau), Kreditinstitute und Versicherungen, Gesundheits-, Veterinär- und Sozialwesen, Abwasser- und Abfallbeseitigung bis hin zur Pflanzenzüchtung und zum Pflanzenbau.

Die Nachfrage nach Anpassungsleistungen wird sich im Bereich der Wirtschaft selbst entwickeln (z. B. in der
Landwirtschaft oder in der Forstwirtschaft), öffentlich (etwa in der kommunalen Daseinsvorsorge) bzw. privat sein (etwa im Gebäudeschutz). Unterstützt wird die Marktentwicklung durch staatliche Regulierung und versicherungswirtschaftliche Interessenlagen. Zeitliche und quantitative Aussagen über mögliche Märkte im Bereich der Klimafolgenanpassung können gegenwärtig noch nicht getroffen werden. Hierfür ist das mögliche Leistungsspektrum zu breit, Aussagen aus der Klimaforschung sind in vielen Fällen noch vage und zukünftige Rahmenbedingungen noch häufig unklar. Dennoch kann davon ausgegangen werden, dass sich das Zeitfenster für den Einstieg der KMU in die Thematik geöffnet hat. Die Aufgabe wird nun darin bestehen, zunächst für ausgewählte Bereiche zukünftige Märkte zu eruieren, was die Abschätzung von Klimafolgen für bestimmte Anpassungsbereiche und die Analyse von Technologieentwicklungen als Basis für innovative Leistungsangebote einschließen muss. Hierbei handelt es sich um ein interdisziplinäres Unterfangen, das den Aufbau entsprechender Kooperationsbeziehungen erfordert.

Technische, marktliche und planungsseitige Unsicherheiten sind nicht nur der Grund, warum sich innovierende Unternehmen zu Netzwerken zusammenschließen, sondern auch dafür, warum sich in frühen Phasen der Entwicklung von Themenbereichen oder auch Technologiefeldern Zentren als Knoten entwickeln, die den organisatorischen und räumlichen Rahmen für die gemeinsame Überwindung solcher 
Unsicherheiten bilden, entsprechende Kompetenzen bündeln und Markt vorbereitend wirken. Aus diesen Gründen wird davon ausgegangen, dass die Nutzung der entstehenden Chancen im Bereich der Klimafolgenanpassung durch den Aufbau eines entsprechenden Innovationszentrums wesentlich befördert werden kann. In einem solchen Innovationszentrum könnten Pilotlösungen sowohl für innovative Anpassungsleistungen als auch für Klimaschutzleistungen in Zusammenarbeit von Forschungseinrichtungen und Unternehmen entwickelt, aus- oder neugegründete Unternehmen angesiedelt und innovative Leistungsangebote demonstriert sowie spezifische Beratungsleistungen angeboten werden.

Im Rahmen des Projekts »Möglichkeiten einer zukünftigen Nachnutzung des Kernkraftwerkstandortes Rheinsberg « im Auftrag der Energiewerke Nord GmbH haben die Autoren des vorliegenden Beitrages empfohlen, ein solches Innovationszentrum für Klimafolgenanpassung aufzubauen. Entsprechende Umsetzungsmaßnahmen wurden im Sommer 2009 eingeleitet.

\section{Literatur}

Bastian Schuchardt et al. (2008): Deutschland im Klimawandel. Anpassung ist notwendig, hrsg. vom Umweltbundesamt.

BMU 2008: BMU-Konferenz »Deutsche Anpassungsstrategie an den Klimawandel « am 15./16.4.2008 in Berlin.

Brandt, M.; Voß, R.; Große, U.; Hartmann, F. (2003): Innovative Technik für Senioren - ein Zukunftsmarkt?, 1. Aufl. - Berlin: News \& Media (Wildauer Schriftenreihe Innovationsmanagement Bd. 3).

Bullmann, U.; Heinze, R. G. (Hrsg.) (1997): Regionale Modernisierungspolitik - Nationale und Internationale Perspektiven, Opladen, 299-316.

Ellwein, T.; Bruder, W. (1982): Innovationsorientierte Regionalpolitik, Opladen.

Fritsch, M. (2003): Von der innovationsorientierten Regionalförderung zur regionalisierten Innovationspolitik, Technische Universität Bergakademie Freiberg, Freiberger Arbeitspapiere 06/2003.

Grünbuch der Kommission der Europäischen Gemeinschaften 2007

Ifm-bonn 2009, www.ifm-bonn.org/index.php.

Koschatzky, K. (2002): Innovationsorientierte Regionalentwicklungsstrategien: Konzepte zur regionalen Technik- und Innovationsförderung. Fraunhofer Institut für Systemtechnik und Innovationsforschung, Karlsruhe, Arbeitspapiere Unternehmen und Region, Nr. R2/2002.

Ott, Hermann E.; Caspar Richter (2008): Anpassung an den Klimawandel - Risiken und Chancen für deutsche Unternehmen, Wuppertal Paper.

Porter, M.E. (1998): Clusters and the new economics of competition. Harvard business review, nov-dec. 1998.
Studie (2003): Studie zur klimatischen Entwicklung im Land Brandenburg bis zum Jahr 2055 und deren Auswirkungen auf den Wasserhaushalt, die Land- und Forstwirtschaft sowie die Ableitung erster Perspektiven, PIK-Report 83.

Umweltbundesamt (2005): Klimawandel in Deutschland. Vulnerabilität und Anpassungsstrategien klimasensitiver Systeme. Forschungsbericht 20141253.

Voß, R. (1996): Innovationsmanagement in kleinen mittelständischen Unternehmen - Ansatzpunkte für ein Forschungskonzept mit Beratungsoptionen. In: Wissenschaftliche Beiträge der Technischen Fachhochschule Wildau, Heft 1/1996, 83-91.

Voß, R. (2005): Ländlicher Raum und Innovation - eine regionalpolitische Daueraufgabe. In: Voß, R. (Hrsg.) (2005): Modernisierung ländlicher Regionen. Wettbewerbsfähigkeit durch Innovation, Bildung, Netzwerke, Berlin.

Zebisch et al. (2005): Klimawandel in Deutschland. (08/05) Forschungsbericht 20141253 UBA-FB 000844.

\section{Autoren}

Prof. Dr. Rainer Voß

TH Wildau [FH]

Forschungsgruppe Innovations- und Regionalforschung Fachbereich Betriebswirtschaft/Wirtschaftsinformatik

Tel. +493375 508-910

rainer.voss@tfh-wildau.de

\section{Dr. Frank Hartmann}

TH Wildau [FH]

Forschungsgruppe Innovations- und Regionalforschung Fachbereich Betriebswirtschaft/Wirtschaftsinformatik Tel. +493375 508-214

frank.hartmann@tfh-wildau.de 